\title{
An Unusual Case of Nasolacrimal Obstruction Caused by Foodstuffs
}

\author{
Hirohiko Matsumoto Akiyo Matsumoto \\ Matsumoto Medical Clinic, Ashiya, Japan
}

\section{Key Words}

Nasolacrimal duct obstruction · Rice grain · Irrigation · Bougie

\begin{abstract}
Here we report a case of nasolacrimal obstruction resulting from rice grains ingested over many years that had formed an obstructive mass. This is a hitherto unreported cause of nasolacrimal obstruction. We exclusively treated the obstruction safely with repeated irrigation with physiological saline. Although there have been various reports on new techniques such as the blind insertion of a bougie and insertion of a silicon tube under endoscopic guidance, successful treatment of this condition is possible with an old-fashioned approach using the time-honored tools of a syringe and an irrigation needle, if utilized patiently and repeatedly. In Japan, we call this 'learning new lessons from the past'. This implies that newer techniques may not necessarily be superior and traditional techniques can offer advantages of safety and simplicity in treating this condition.

(c) 2015 S. Karger AG, Basel
\end{abstract}

\section{Introduction}

Tear fluid drains through the lacrimal punctum into the lacrimal canaliculi; it then flows through the lacrimal sac and the nasolacrimal duct before being absorbed by the nasal mucosa. When this pathway becomes blocked, the resulting condition is known as nasolacrimal duct obstruction, which may be either congenital or acquired [1]. Over the last 15 years, our clinic has experienced 64 cases of nasolacrimal duct obstruction, all of which were successfully treated with lacrimal passage irrigation. In this procedure, $0.4 \%$ xylocaine ophthalmic solution is injected using a $1-\mathrm{ml}$ syringe tube and a lacrimal passage irrigation needle, with the tip of the needle being inserted into the inferior lacrimal punctum pointing toward the nose and facing slightly downward. To facilitate irrigation, the patient is asked to snort in order to create negative pressure in the nasal cavity. Once the patient has registered the

\section{KARGER 125/3 $\quad \begin{aligned} & \text { Hirohiko Matsumoto, MD, PhD } \\ & 13-163 F, \text { Sanjo-Minami }\end{aligned}$ \\ Ashiya 659-0086 (Japan) \\ E-Mail hirohiko@gol.com}


Matsumoto and Matsumoto: An Unusual Case of Nasolacrimal Obstruction Caused by Foodstuffs

bitter taste of the xylocaine, irrigation should be performed repeatedly with physiological saline. In these 64 cases, we performed at least 10 irrigations for each patient, and for some, we performed 50 or more. Of the 64 cases, recurrence only occurred in the 1 case that we present here. In this patient, the blockage resulted from rice grains ingested over a period of many years, forming an obstructive mass. This phenomenon has previously been unreported as a cause of nasolacrimal obstruction [2].

\section{Case Presentation}

The patient, a 39-year-old male who works as a surgeon and is the lead author of this report, experienced abnormal lacrimation of the left eye while at work. He underwent irrigation performed by the second author, who is an ophthalmologist. However, the condition recurred 5 times during the following 2 years despite repeated irrigation on each occasion. During the sixth treatment, after approximately 20 irrigations had been completed, an object was suddenly ejected into the rear of the patient's nasal cavity. On histological examination, this object was found to be an agglomeration of rice grains (fig. 1, fig. 2) and was believed to have penetrated the nasolacrimal duct over a long period via reflux through Hasner's valve. This mass then became molded into a rectangular, cone-shaped plug, matching the shape of the nasolacrimal duct. Since this successful treatment, there has been no further recurrence. Approximately 1 month after completing treatment, the author underwent detailed examination of the paranasal sinuses by an otolaryngologist using fiberscopy. Although it was not possible to view up to Hasner's valve, no other abnormalities were noted within the range visible by fiberscopy.

\section{Discussion}

To the best of our knowledge, there have been no other reported cases of nasolacrimal duct obstruction caused by the retention of foodstuffs [2]. The patient had a habit of talking while eating, and we concluded that a rice grain had at some point penetrated the orifice of the nasolacrimal duct from the nasal cavity side via reflux through Hasner's valve. This led to incomplete valve closure and allowed the subsequent entry of a succession of rice grains, coalescing into a single mass and causing the blockage. In this case, the nasolacrimal duct appears to have been nearly completely obstructed by a lump of rice. During initial treatment, neither anesthetics nor physiological saline solution could pass through the duct, instead pouring out of the inferior punctum. In the latter phase of treatment, as the lump of rice started to slowly drop down into the paranasal sinus, water was gradually able to pass through the site despite meeting some resistance. When the lump of rice emerged from the paranasal sinus, this resistance to passing physiological saline solution disappeared and the patient suddenly felt physiological saline solution flow into the paranasal sinus. Backflow caused the rice grain to initially enter the nasolacrimal duct, after which deposits built up over a number of years to cause an obstruction. We then started to treat this with flushing, which could not have caused the backflow considering the chronology, because there is no possibility that backflow occurred as a result of flushing. Nasolacrimal duct obstruction is typically attributed to the accumulation of ocular discharge within the lacrimal passages; however, such cases may be more common, although they may go unrecognized [3].

In the treatment of nasolacrimal duct obstruction, a bougie is normally inserted immediately after an irrigation test. Because this blind insertion can be dangerous, many institu- 
Matsumoto and Matsumoto: An Unusual Case of Nasolacrimal Obstruction Caused by Foodstuffs

tions perform this operation under endoscopic guidance [4]. However, we believe that successful treatment is possible using only repeated irrigation with physiological saline. This approach is safe and simple, allows flushing of the entire lacrimal passage, and is effective in preventing recurrences. To perform this procedure, the physician should wait for the water to completely pass through during the irrigation test. Then, instead of immediately inserting a bougie, irrigation should be patiently repeated numerous times. Therefore, the irrigation should be considered not only as a test but also as a form of washout therapy.

\section{Conclusions}

The patient reported here first presented with persistent lacrimation. Only after repeated unsuccessful treatments was it determined to be due to obstruction by impacted foodstuffs. We have never observed any other cases in which a lump of foodstuff was retained in the nasolacrimal duct and caused an obstruction, as in the present patient. In other cases, the discharge contained in tears adhered to the lumen of the nasolacrimal duct and caused either narrowing or a state close to obstruction. Therefore, such cases could be treated using flushing performed repeatedly to gradually wash out the discharge. This is not a frequent cause of nasolacrimal obstruction, but it should perhaps be considered as a possible factor in similar cases of refractory nasolacrimal disorders.

\section{Acknowledgement}

The authors would like to thank Enago (www.enago.jp) for the English language review.

\section{Statements of Ethics}

Written informed consent was obtained from the patient.

\section{Disclosure Statement}

The authors declare no conflict of interest.

\section{References}

1 Mills DM, Meyer DR: Acquired nasolacrimal duct obstruction. Otolaryngol Clin North Am 2006;39:979-999.

-2 Tucker N, Chow D, Stockl F, Codère F, Burnier M: Clinically suspected primary acquired nasolacrimal duct obstruction: clinicopathologic review of 150 patients. Ophthalmology 1997;104:1882-1886.

-3 Owji N, Abtahi SMB: Does gastroesophageal reflux contribute to development of acquired nasolacrimal duct obstruction? Med Hypotheses 2010;74:455-456.

4 Moscato EE, Dolmetsch AM, Silkiss RZ, Seiff SR: Silicone intubation for the treatment of epiphora in adults with presumed functional nasolacrimal duct obstruction. Ophthal Plast Reconstr Surg 2012;28:35-39. 


\section{Case Reports in \\ Ophthalmology}

\begin{tabular}{l|l}
\hline \multicolumn{2}{l}{ Case Rep Ophthalmol 2015;6:307-310 } \\
\hline DOI: 10.1159/000439425 & $\begin{array}{l}\text { @ 2015 S. Karger AG, Basel } \\
\text { www.karger.com/cop }\end{array}$ \\
\hline
\end{tabular}

Matsumoto and Matsumoto: An Unusual Case of Nasolacrimal Obstruction Caused by Foodstuffs

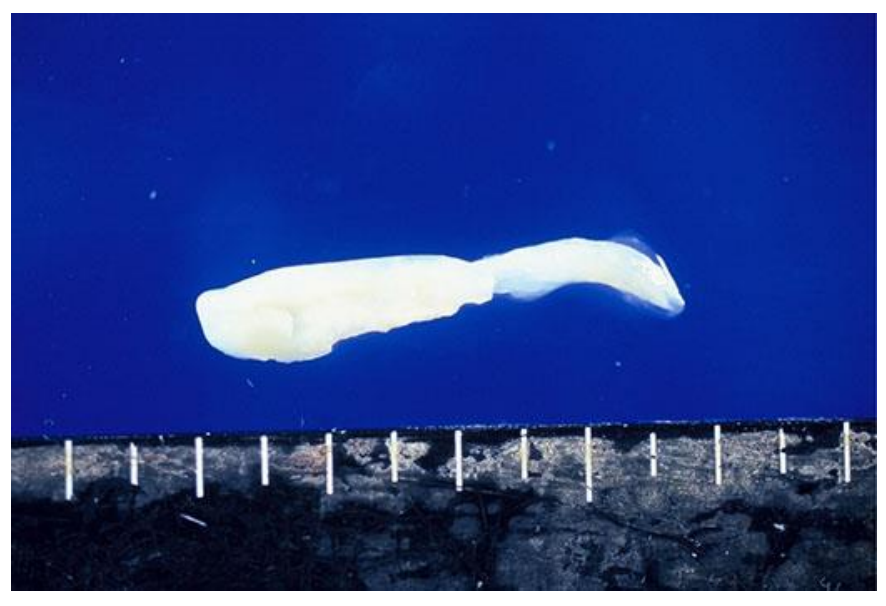

Fig. 1. Cone-shaped mass obstructing the lacrimal duct.

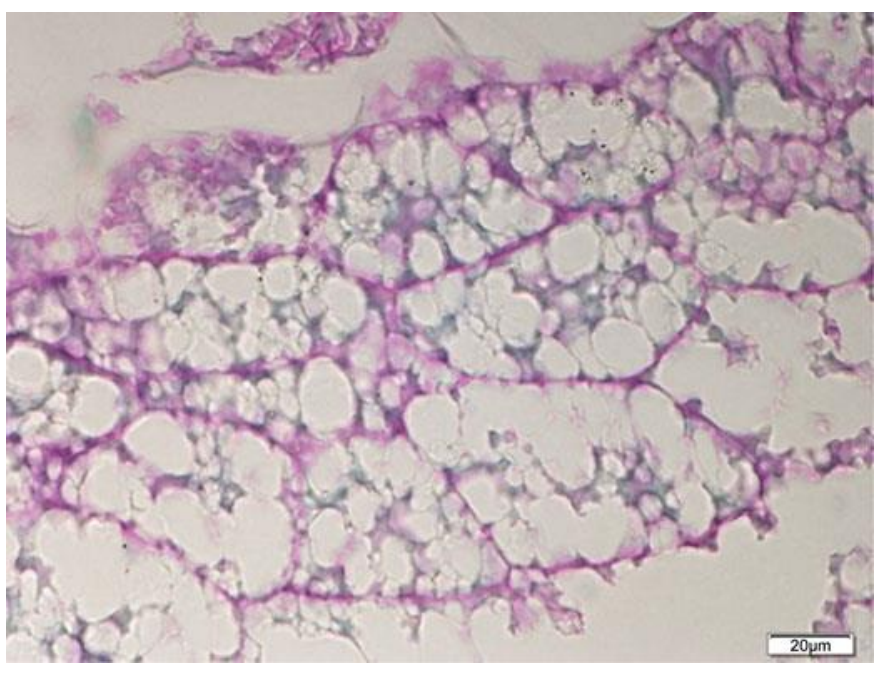

Fig. 2. Image from the histological analysis. 